\section{VIOLENCIA SIMBÓLICA EN LA EDUCACIÓN FÍSICA ESCOLAR: UN ANÁLISIS CRÍTICO DE LAS EXPERIENCIAS NEGATIVAS DEL FUTURO PROFESORADO DE EDUCACIÓN PRIMARIA}

\author{
VIOLÊNCIA SIMBÓLICA NA EDUCAÇÃO FÍSICA ESCOLAR: UMA ANÁLISE \\ CRÍTICA DAS EXPERIÊNCIAS NEGATIVAS DO FUTURO DOCENTE DE \\ EDUCAÇÃO PRIMÁRIA
}

SYMBOLIC VIOLENCE IN SCHOOL PHYSICAL EDUCATION: A CRITICAL ANALYSIS OF THE NEGATIVE EXPERIENCES OF FUTURE TEACHERS IN PRIMARY EDUCATION

Maria José Camacho-Miñano*, María Prat Grau*
Palabras clave:

Docentes.

Narrativa.

Violencia.

Masculinidad.

\begin{abstract}
Resumen: Este estudio profundiza sobre las experiencias negativas de la Educación Física escolar cursada por el futuro profesorado de Educación Primaria, utilizándose para el análisis el concepto de violencia simbólica de Bourdieu. Se ha realizado un análisis de 155 narrativas de estudiantes de grado de Educación Primaria en dos universidades españolas. Los resultados muestran que la violencia simbólica se ejerce por la inadecuación de las identidades físicas de este alumnado a los discursos del rendimiento, que son, a su vez, legitimados por el profesorado y otros estudiantes exitosos. Este tipo de violencia oculta se proyecta en las identidades físicas y profesionales del futuro profesorado.
\end{abstract}

Palavras-chave: Docentes. Narrativa. Violência. Masculinidade.

Keywords: Teachers. Narrative. Violence. Masculinity.
Resumo: Este estudo aprofunda as experiências negativas da Educação Física Escolar cursada pelo futuro docente de Educação Primária, usando o conceito de violência simbólica de Bourdieu para a análise. Foi realizada uma análise de 155 narrativas de estudantes de graduação em Educação Primária de duas universidades espanholas. Os resultados indicam que a violência simbólica é exercida pela inadequação das identidades físicas dos graduandos aos discursos do rendimento, os quais, por sua vez, são legitimados pelos docentes e por estudantes exitosos. Este tipo de violência subliminar projeta-se também nas identidades físicas e profissionais do futuro docente.

Abstract: This is an in-depth study into future Primary Education teachers' negative experiences in Physical Education School, using Bourdieu's concept of symbolic violence. An analysis was conducted of 155 narratives of Primary Education teacher students in two Spanish universities. The results show that symbolic violence is exercised through the inadequacy of those students' physical identities to performance discourses, which, in turn, are legitimized by teachers and other successful students. This hidden violence is projected onto their physical and professional identities as future teachers.
*Universidad Complutense de Madrid. Madrid, España.

E-mail: mjcamacho@edu.ucm.es; *Universidad Autónoma de Barcelona. Cataluña, España.

E-mail: maria.prat@uab.cat

Recebido em: 26-12-2017 Aprovado em: 29-06-2018

DOI: https://doi.org/10.22456/1982-8918.79171 (c) (1) (8) Licence 


\section{INTRODUCCIÓN}

El objetivo del presente estudio es desvelar la Violencia Simbólica (VS) presente en las experiencias negativas de la Educación Física (EF) escolar rememorada por el futuro profesorado de Educación Primaria y su impacto sobre su identidad personal y pedagógica actual. Se adopta para ello un enfoque narrativo que visibiliza los discursos hegemónicos de la EF y las relaciones de poder inscritas en las historias individuales del futuro profesorado, a través del análisis de las relaciones que existen entre la experiencia subjetiva y las estructuras sociales (DEVÍS-DEVÍS, 2017). La noción de VS de Bourdieu (2000) se utiliza para analizar críticamente las vivencias negativas del alumnado que ha sufrido la marginación y exclusión por carecer de las disposiciones que se valoran en la asignatura y que, paradójicamente, suele ser cómplice de su propia sumisión. El análisis de los discursos de la EF (LÓPEZ PASTOR; GEA FERNÁNDEZ, 2010; TINNING, 1996; 2010), y específicamente de los discursos hegemónicos, permite comprender el cuerpo y disposiciones que se legitiman en la asignatura.

Aunque los estudiantes acceden al Grado de Maestro en Educación Primaria con una carga heterogénea de vivencias y experiencias en torno a la EF (GARRETT; WRENCH, 2007; GONZÁLEZ PLATE; RIVERA; TRIGUEROS, 2014), seguimos observando en nuestro alumnado una gran presencia de experiencias negativas. Este tipo de experiencias son particularmente problemáticas puesto que generan en el futuro profesorado una preparación inadecuada y una baja confianza para enseñar la asignatura (GARRETT; WRENCH, 2007; MORGAN; BOURKE, 2008). La necesidad de profundizar en su estudio y de considerarlas en la formación universitaria se justifica por la estrecha interrelación que existe entre lo personal y lo pedagógico (FERNÁNDEZ-BALBOA, 1998), convirtiéndose estas experiencias previas en la lente o filtro a través del cual el profesorado en formación negocia y da sentido a su aprendizaje universitario (GARRETT; WRENCH, 2007). De esta forma, la VS que ha sufrido este alumnado en la EF recibida, según recogen en la interpretación que realizan de estas experiencias en sus narrativas, podría impregnar la propia conceptualización de la EF y su valor educativo como futuro profesorado de Educación Primaria, motivo que justifica su estudio como clave para nuestra práctica docente e investigadora.

\subsection{Violencia simbólica en la EF Escolar}

Bourdieu describe la violencia simbólica como aquella forma de "violencia que se ejerce contra un agente social con su complicidad"(BOURDIEU; WACQUANT, 2005, p.240). Es una violencia que no opera a través de la coacción o violencia explíita, sino a través de un poder legitimador en el que los dominados aceptan como legítima su propia condición de dominación (BOURDIEU; WACQUANT, 2005). Se trata de una forma de "persuasión oculta" por la que las personas que ocupan las posiciones dominadas reproducen las categorías de percepción y valoración que son la condición misma de su dominación, convirtiéndose en cómplices de la dominación a la que están sometidas (BOURDIEU; WACQUANT, 2005; FERNÁNDEZ, 2005). Este poder simbólico funciona mediante la falta de reconocimiento de su carácter fundamentalmente arbitrario, que se ejerce de forma implacable por lo que se considera el "orden de las cosas", lo que es "de sentido común" (FERNÁNDEZ, 2005). Su funcionamiento se apoya en disposiciones previamente constituidas, entendidas estas como una manera duradera de ser o hacer que se encarna en los cuerpos (habitus) y que llevan a percibir, pensar 
y comportarse de una cierta manera en el espacio social (BOURDIEU; WACQUANT, 2005; FERNÁNDEZ, 2005).

Dentro del ámbito de la EF, diversos estudios han analizado la VS que existe en la propia conceptuación de la habilidad motriz, que ha de entenderse como una construcción social cuya apreciación depende de la capacidad para rendir en aquellas actividades que se valoran en el campo de la EF y que determinan el capital simbólico de la asignatura (HAY; LISAHUNTER, 2006; WRIGHT; BURROWS, 2006). Específicamente, se reconocen las disposiciones asociadas a los discursos del rendimiento y en particular al deporte, por lo que esta conceptualización de habilidad motriz no es neutral sino que se asocia con las formas hegemónicas masculinas (BELTRÁN-CARRILLO; DEVÍS-DEVÍS; PEIRÓ-VELERT; BROWN, 2012; GARRETT; WRENCH, 2007; REDELIUS; FAGRELL; LARSSON, 2009; WRIGHT; BURROWS, 2006).

A través de este privilegio de las habilidades deportivas y la competición como atributos valorados, las personas bien posicionadas dentro del campo (chicos deportistas y profesorado de EF), son agentes reproductores que legitiman la naturaleza de esa habilidad para mantener su poder (HAY; LISAHUNTER, 2006). Y aquel alumnado con falta de habilidad física, poca condición física o un cuerpo inadecuado, termina asumiendo que ellos y ellas son el problema, "[...] y acaban perdiendo su autoestima y dignidad, aceptando esta VS con sumisión, tristeza y dolor" (BELTRÁN-CARRILLO et al., 2012, p. 21) e incluso miedo (MONFORTE; PÉREZSAMANIEGO, 2017), llegando a autoexcluirse de otros contextos de práctica de actividad física (BELTRÁN-CARRILLO et al., 2012).

\subsection{Los discursos de la Educación Física Escolar}

El término discurso remite a las formas de pensar y hablar sobre aspectos de la realidad, lo que permite construir y dar significado a nuestra visión del mundo. Los discursos incluyen además todas las prácticas sociales, formas de subjetividad y relaciones de poder intrínsecas (GARRETT; WRENCH, 2007) ya que aquellos discursos dominantes, legitimados y transmitidos a través de instituciones como la escuela, presentan particulares formas de pensar como las "verdaderas", convirtiéndose en vehículos para la ideología y el control social, generando también significados para configurar las propias identidades (KIRK, 1992). Salvando la complejidad de este concepto y de sus intentos de clasificación, en EF se han identificado dos grandes tipos de discursos: los orientados al rendimiento y los orientados a la participación (LÓPEZ PASTOR; GEA FERNÁNDEZ, 2010; TINNING, 1996, 2010).

Dentro de los discursos orientados al rendimiento, destaca el deporte como contenido que sigue predominando en el currículum escolar. Esta EF "deportivizada" promueve la adquisición de técnicas y habilidades propias de los deportes, en los que las actividades se estructuran en torno a la competición y se tiende a potenciar al desarrollo de atributos masculinos (p.e., fuerza, agresividad). Asociado a este discurso se generan prácticas fundamentadas en el individualismo, la jerarquía y el elitismo y privilegio de los ganadores, junto con la consecuente marginación y exclusión del resto de estudiantes (BELTRÁN-CARRILLO et al., 2012; TINNING, 1996, 2010)

Por otra parte, el creciente interés de la EF por el discurso de la salud orientado a la promoción de estilos de vida activos, ha promovido erróneamente un resurgir de los test de 
condición física (LÓPEZ-PASTOR;KIRK; LORENTE-CATALÁN; MACPHAIL; MACDONALD, 2013), que a menudo se utilizan como base para la calificación, reproduciendo la ideología propia de los discursos del rendimiento. Además, el parámetro de referencia del cuerpo sano y en forma coincide de forma simplista con el ideal estético actual, motivando que en EF surjan nuevas jerarquías del cuerpo en relación al tamaño, forma y peso corporal (EVANS; RICH; ALLWOOD; DAVIES, 2007). La EF según este tipo de discursos se define en base a los capitales físicos de tener un cuerpo fuerte, musculoso y en forma, ser físicamente activo y hábil en los deportes, junto con disposiciones instrumentales para el éxito deportivo, como ser competitivo y agresivo (WRIGHT; BURROWS, 2006). Esta forma dominante de masculinidad encarna al estudiante de EF ideal, que a su vez se construye relacionalmente frente a otras masculinidades subordinadas y las feminidades, legitimando ideológicamente la dominación masculina y el patriarcado (BELTRÁN-CARRILLO et al., 2012; FERNÁNDEZ, 2005).

Por el contrario, los discursos de la participación tienen, como su nombre indica, el propósito de incrementar la participación en la cultura del movimiento (LÓPEZ PASTOR; GEA FERNÁNDEZ, 2010; TINNING, 1996, 2010). Se trata de una EF con un enfoque inclusivo y comprensivo, en la que se busca que todo el alumnado pueda experimentar su cuerpo y movimiento de forma satisfactoria. Por ello, se da especial importancia a lo vivencial, lo expresivo, la toma de conciencia motriz y corporal, lo grupal y lo colaborativo, facilitándose la diversidad y la experimentación motriz (LÓPEZ PASTOR; GEA FERNÁNDEZ, 2010). Esta orientación exige romper con el cuerpo-objeto, el cuerpo genérico sustentado por el discurso técnico de la EF, para centrarse en el cuerpo-sujeto y su identidad diferencial (VICENTE, 2013). Paechter(2003) ha señalado que las danzas expresivas y creativas y las actividades en la naturaleza con carácter cooperativo son los contenidos que podrían promover la vivencia, los procesos y el valor comunicativo del cuerpo, revalorizando estos capitales físicos vinculados con la feminidad.

Los discursos hegemónicos en la EF han sido y siguen siendo los discursos del rendimiento, que promueven la noción de "cuerpos valorados", es decir, cómo ciertos cuerpos, tanto por su apariencia física como por su habilidad motriz, tienen un mayor estatus o consideración en la EF (AZZARITO, 2009; WRIGHT; BURROWS, 2006). En este contexto aquellas personas que no encajan con los modelos hegemónicos, rememoran vivencias en EF identificadas como negativas, y que pueden entenderse como formas de VS cuando no se reconocen las estructuras sociales que les dotan de significado.

\section{METODOLOGÍA}

En este estudio han participado 155 estudiantes (131 mujeres y 24 hombres) que cursaban Grado de Maestro de Educación Primaria en dos universidades españolas, en Cataluña y Madrid. La investigación se ha desarrollado en el contexto de la asignatura "EF y su Didáctica", impartida por dos profesoras (y autoras del trabajo) a cuatro grupos de clase durante los cursos 2013-2014 y 2014-2015. La asignatura se imparte con carácter obligatorio y duración semestral en segundo curso del Grado en ambas universidades. Los datos empíricos se recogieron a través de la realización de una narrativa, en forma de relato autobiográfico escrito (BOLÍVAR; DOMINGO; FERNÁNDEZ, 2001), centrada en sus experiencias previas en la EF. Para ello los participantes respondieron por escrito a una serie de preguntas abiertas, 
centradas en identificar contenidos y actividades más significativas, sus mejores y peores experiencias en la asignatura, así como los sentimientos asociados y su valoración sobre el impacto de la EF recibida. Para garantizar el carácter voluntario de la participación, se les invitó a enviar de forma individual sus relatos a la plataforma virtual de cada grupo (Participaron 155 personas, un $73.8 \%$ del total de 210 estudiantes). El anonimato se garantizó asignando un código de identificación a cada relato.

El análisis de los datos comenzó con una lectura inicial de los textos y la selección de aquellos centrados en vivencias negativas. Posteriormente, se realizó un diseño de categorías y temas, que se ha configurado de forma mixta con apoyo del programa NVIVO v.10. La primera sistematización de aspectos significativos y dominantes de los datos se realizó con codificación inductiva o abierta (GLASER; STRAUSS, 1967), mediante continuas comparaciones de los códigos y la identificación de similitudes, diferencias y relaciones entre ellos. Posteriormente, se realizó una codificación más teórica, que permitió identificar los temas principales a partir de los conceptos clave y en función del propósito del estudio. Ambas autoras participaron en la codificación y debatieron sobre las categorías identificadas hasta alcanzar el consenso.

\section{RESULTADOS Y DISCUSIÓN}

\subsection{Violencia simbólica por la inadecuación de identidades físicas a los discursos del rendimiento.}

La rememoración de experiencias negativas es significativa y mayoritaria cuando las participantes en este estudio, en su mayoría chicas, hacen referencia a la EF recibida en Educación Secundaria, en la que predominan los discursos del rendimiento. Numerosos ejemplos señalan los "deportes de equipo con una gran carga de competitividad" como los contenidos predominantes:

Tengo recuerdos más negativos que positivos, dado que yo no era especialmente buena para los deportes que solíamos realizar como: fútbol, baloncesto, hockey, etc. Las clases se basaban en competir unos con otros, y donde los grupos los formaban los dos mejores de la clase, siempre dejando para el final a los alumnos que nadie quería en su equipo. Básicamente, la asignatura potenció en mí sentimientos como: inferioridad, miedo al ridículo o incluso en algunos casos me sentía excluida del grupo a la hora de formar equipos para competir (M135).

El enfoque tradicional en la enseñanza de este tipo de contenidos deportivos potencia la competición y el despliegue de fuerza, poder y agresividad, valores que celebran la masculinidad hegemónica y que refuerzan a determinados grupos de chicos, marginando y excluyendo al resto (BELTRÁN-CARRILLO et al., 2012; LARSSON; FAGRELL; REDELIUS, 2009). En los datos también resultan significativas las pruebas de condición física, especialmente los test de resistencia que, al igual que en otros estudios (WRENCH; GARRETT, 2008), son vivenciados con angustia y dolor por aquel alumnado cuyo rendimiento no alcanza los estándares establecidos: "Tenía la sensación que me estaba asfixiando y que no llegaría a realizar las vueltas mínimas para aprobar. Por culpa de esta presión y tensión sufrí un ataque de ansiedad" (M4).

Se trata de comportamientos visibles y cuantificables que ponen de manifiesto el ideal físico-deportivo del cuerpo-máquina cuya eficiencia refuerza el poder de los discursos biológicos, la naturalización de las diferencias y la jerarquía y, en definitiva, son una muestra 
de la cultura de performatividad que celebra la comparación, la medición, la evaluación y la responsabilidad individual (EVANS et al., 2007)y en la que se establece lo masculino como el referente para el máximo rendimiento (BELTRÁN-CARRILLO et al., 2012).

En estos discursos, la noción de competencia resulta clave para entender cómo los estudiantes se identifican a sí mismos como poco competentes para la EF debido a que no tienen las disposiciones que se requieren y valoran en la asignatura. Multitud de expresiones reflejan en los relatos cómo los estudiantes tienen interiorizado la vivencia de sus identidades físicas como una carencia: "yo era mala", "era de las peores de la clase" "nunca he sido muy coordinada con los pies", "no tenía una condición física adecuada", etc. De esta forma, las personas "menos hábiles" o "poco competentes" se hacen responsables individualmente de su falta de capacidad, sin tener en cuenta la existencia de estructuras sociales que valoran precisamente aquellas capacidades corporalizadas para rendir en estas formas de habilidad legitimadas en el campo de la EF (BARBERO, 2007; HAY; LISAHUNTER, 2006; WRIGHT; BURROWS, 2006).

El carácter competitivo de la EF genera en aquel alumnado con bajo nivel de habilidad deportiva una sensación de impotencia y falta de control en este contexto relacional en el que se sienten inferiores y, en algunos casos, excluidos. Incluso hay relatos de miedo y pánico a hacerse daño o sufrir cuando se demandaban niveles de exigencia física muy por encima de las propias posibilidades motrices:

Sinceramente me ponía muy nerviosa, tenía miedo y sufría porque no quería hacerlo mal y que los compañeros se rieran de mí. Sobre todo cuando me tocaba saltar, lo pasaba realmente mal, me sudaban las manos, temblaba,... lo recuerdo como algo muy negativo (M108).

Como señalan Monforte y Pérez-Samaniego (2017, p.96), se trata de una VS "[...] articulada en términos invisibles, amortiguada, porque se apoya en la complicidad muda del propio cuerpo" y que se produce en el momento en que el alumnado se hace responsable de sus emociones, sin ser consciente de las estructuras sociales que le dan significado.

Ante esta situación, algunas estudiantes, en su mayoría chicas, relatan cómo trataban de compensar su falta de rendimiento motriz a través de lo cognitivo, mediante buenas calificaciones en exámenes teóricos. Según Hay y Macdonald(2010) esta realidad refuerza la posición de género subordinada de estas estudiantes que carecen del cuerpo legitimado en EF, y que se ven forzadas a compensar este "déficit" mediante atributos no específicos del campo.

Como lo pasaba mal en las evaluaciones de los ejercicios prácticos y, debido a mi altura y condición física, algunos de ellos me costaban, me esforzaba mucho y estudiaba para sacar buenas notas en el examen teórico para salvar la asignatura. Lo que me provocaba mucha angustia a lo largo del curso (M129).

Otras estudiantes, todas ellas mujeres, explican cómo aprendieron a desarrollar estrategias de evitación de las clases ("descuidar el equipamiento en casa, encontrarse mal, simular lesiones, notas en la agenda de los padres, etc."). Esta autoexclusión podría ser una forma de resistencia para proteger su identidad femenina frente a la cultura masculina que promueve la EF (COCKBURN; CLARKE, 2002).

Esta inadecuación de las identidades físicas está estrechamente ligada a la percepción de que la propia apariencia física no se ajusta a los parámetros del cuerpo que se considera "ideal y deseable" para el rendimiento deportivo (AZZARITO, 2009; GARRETT, 2004). EI 
impacto de este tipo de situaciones sobre la identidad es considerable porque, a diferencia de la relativa privacidad del aprendizaje en el aula, en las clases de EF existe una exposición pública del cuerpo, que queda sujeto a la observación y vigilancia de otros estudiantes y del profesorado (FISETTE, 2012). Se acentúan así los sentimientos de vergüenza, miedo al ridículo, ansiedad y culpa por no alcanzar los niveles requeridos por este modelo corporal reconocido como legítimo.

Mi peso era elevado en relación con mi estatura, es decir, no me encontraba en buenas condiciones físicas (...) y en las pruebas prácticas, me sentía bastante humillada. Vivencié un ciclo de burlas constantes por parte de mis compañeros. Entonces, me sentía tan mal con mi misma, y especialmente con mi aspecto físico, que sufrí una grave enfermedad que me costó mucho de superar [...]. (M60).

Al igual que los resultados de otros trabajos (FISETTE, 2012; GARRETT, 2004) son fundamentalmente las chicas las que muestran de forma explícita una intensa preocupación por su apariencia física. El vestuario, el momento de las duchas o cambiarse de ropa, así como las clases de natación, son situaciones particularmente críticas ya que el cuerpo queda visiblemente expuesto y se convierte en objeto de evaluación y de comparación (FISETTE, 2012). En definitiva, dentro de los discursos del rendimiento, se van configurando cuerpos fracasados (HAY; LISAHUNTER, 2006; WRIGHT; BURROWS, 2006), que tienen una baja consideración dentro de este contexto social de la clase de EF y que lo son porque hay otras identidades (profesorado y estudiantes exitosos) que para mantener su poder, legitiman y reproducen estos discursos hegemónicos.

\subsection{Violencia simbólica y real ejercida por otros estudiantes y el profesorado}

Numerosos ejemplos ilustran que las actividades competitivas promueven relaciones negativas entre los iguales, debido a los comportamientos dominantes, competitivos y agresivos de algunos estudiantes. Este despliegue de masculinidad hegemónica lleva asociado el desprecio y humillación del alumnado menos habilidoso, que aparece en los datos como objeto de burlas, comentarios negativos y agresiones verbales, e incluso físicas:

\footnotetext{
Otras vivencias que recuerdo como especialmente negativas son los momentos donde durante el juego o el partido que estábamos haciendo en grupos, fallar en algún pase o toque que hacía perder al equipo. En estos momentos, las reacciones de los alumnos más competitivos eran la de despreciar mediante comentarios negativos que afectaban. En mi caso me afectaban mucho y me hacían sentir inferior a los demás (M126).
}

Las narrativas recogen multitud de escenas en las que el deseo de ganar parece justificar este tipo de comportamientos por parte de los chicos dominantes, como señalan otros estudios (BELTRÁN-CARRILLO et al., 2012; GARRETT; WRENCH, 2007; LARSSON et al., 2009). Junto con la violencia real (tanto verbal como física), estos ejemplos de discriminación pueden considerarse poderosas formas de VS ya que "enmascaran y refuerzan las relaciones de poder corporalizadas de la jerarquía de habilidad física y al hacerlo recuerdan a las víctimas que no encajan en esta cultura" (BELTRÁN-CARRILLO et al., 2012, p. 20). De esta forma, en la EF rememorada por los estudiantes a profesorado se normaliza la relación entre comportamientos agresivos, violencia simbólica y real y dominación masculina (BELTRÁN-CARRILLO et al., 2012; BROWN, 2005). 
La jerarquía de los cuerpos se hace explícita al permitir a los estudiantes formar equipos, práctica que conforma un poderoso currículum oculto a través del cual aprenden de forma implícita la relación dominador/dominado (BOURDIEU, 2000). Como en otros trabajos (GARRETT; WRENCH, 2007; GONZÁLEZ PLATE et al., 2014), el hecho de no ser elegido revela poderosos discursos de incompetencia y fracaso, asociados a emociones negativas que perduran más allá de la situación concreta. En este caso, es clara que la ausencia de capital físico deriva en la imposibilidad de acceder al capital social al que otros estudiantes sí tienen acceso, con el riesgo de ser excluido.

[...] Los dos capitanes, a menudo siempre los mismos (parecía que lo eran por el derecho divino, sintiéndose superior al resto) escogían por turnos a las personas que formarían parte de su equipo [...] En alguna ocasión me quedaba de las últimas, bajo la atenta mirada del resto de compañeros/as, conscientes que si me quedaba la última, era porque "nadie me quería", y esto representaba una losa (M100).

El profesorado se revela en los datos como agente clave que ejerce VS. Por un lado, se advierte una ausencia de intervención del profesorado en las situaciones descritas de dominancia de (algunos de) los chicos, ya que se considera "algo normal o natural y que se debe manejar logísticamente en lugar de desafiarse" (LARSSON et al., 2009,p.1). Además, el profesorado refuerza y presta más atención al alumnado competente en este modelo androcéntrico de $E F$, generalmente chicos y que practican deporte extraescolar:

La mayoría de maestros que tuve, potenciaban la competición, premiando siempre a los buenos de la clase y dejando en evidencia a los alumnos que no se les daba bien, con comentarios delante todo el grupo (M135).

No me gustaba nada que los profesores parecían tener favoritismo con aquellos que participaban en el cros o hacían algún tipo de actividad extraescolar como atletismo y baloncesto (M129).

De esta forma el profesorado, fuertemente influenciado por el ámbito deportivo, transmite activamente a esta nueva generación de estudiantes lo que tienen interiorizado como sus propios valores y prácticas masculinas (BROWN, 2005). Las creencias del profesorado están inscritas en poderosos discursos de género en los que se considera que las chicas están menos capacitadas que los chicos para rendir en la asignatura, lo que perpetúa e institucionaliza la organización jerárquica de género.

En secundaria tuvimos durante un tiempo un profesor que valoraba más los resultados de los niños que de niñas. Un día nos cronometraba haciendo sprint y quedé tercera en la lista mixta entre niños y niñas y sorprendió al profesor que los primeros no fueran todo niños (M126).

También existen datos que apuntan a que el profesorado ejercía en ocasiones una violencia explícita, a través de agresiones verbales como las relatadas: "me hacía sentir fatal con sus palabras" "me humillaba", "me gritaba", "me decía que me pesaba el culo", etc.

Pero el profesorado destaca principalmente como agente que institucionaliza la VS, en cuanto que es responsable de la selección del currículum deportivo mayoritario, adopta un enfoque técnico en su enseñanza y prioriza sistemas de evaluación basados en la cuantificación y clasificación. En este sentido, los test de condición física no valoran la capacidad de aprender sino que reconocen las particulares disposiciones que ya tienen los individuos dentro de los discursos hegemónicos, que se perpetúan y se aceptan como verdades incuestionables (WRIGHT; BURROWS, 2006). 
Tuve un mal profesor de EF y solo nos valoraba dependiendo de cuánto podías correr, cuanto peso podías levantar o cuánta elasticidad tenías (en mi caso no era muy elástica). Pienso que no es una buena forma de evaluarnos porque no se tenían en cuenta nuestro progreso, sino la forma física que se tenía (M135).

Las calificaciones objetivan y hacen pública las desigualdades y jerarquías que previamente solo existían de forma implícita, convirtiéndose en formas de poder simbólico (FERNÁNDEZ, 2005) que institucionalizan la valía de cada estudiante. Se generan así formas de VS, en la que los estudiantes que se perciben y son percibidos por otras personas significativas como con menor nivel de habilidad son, de forma más o menos explícita, marginados y excluidos en una EF orientada al rendimiento (HAY; LISAHUNTER, 2006).

Todo ello configura VS porque implícitamente excluye del campo de la EF al alumnado que carece de las disposiciones hegemónicas que se valoran en la asignatura. Este tipo de EF orientada al rendimiento, no es neutral, sino que perpetúa las relaciones de género tradicionales que reproducen la dominación masculina (BELTRÁN-CARRILLO et al., 2012; BOURDIEU, 2000).

\subsection{Consecuencias de la vs sufrida en la ef sobre la identidad personal y pedagógica del profesorado}

Las experiencias negativas de la EF cursada han tenido un impacto negativo, ya que algunas personas se posicionan como sujetos físicamente inactivos, o activos a pesar de la EF cursada y de su profesorado, produciéndose el fenómeno de que la asignatura promueve paradójicamentela inactividad (BELTRÁN-CARRILLO et al., 2012):

\footnotetext{
El impacto de la EF que recibí en el colegio sobre mi vida actual no es muy significativo, a causa de la mala suerte que he tenido con mis profesores de EF. Algunos compañeros tienen la misma opinión que yo: padres, amigos y actividades extracurriculares nos han ayudado a interesarnos en el deporte. Mi experiencia personal es que empecé a motivarme cuando me apunté al gimnasio, porque encontré un monitor [...]. (M121).
}

El recuerdo de estas experiencias negativas suscita en el alumnado "nerviosismo", "presión" e incluso "incredulidad" al tener que cursar una asignatura vinculada con la enseñanza de la EF en su formación universitaria; incluso hay quien señala explícitamente que esta formación "no debería ser obligatoria". Por otra parte, aunque hay estudiantes que realizan su narrativa de la EF sin una reflexión sobre sus consecuencias pedagógicas, también hay quien cuestiona la EF recibida y su profesorado, planteando la necesidad de un modelo alternativo a los discursos hegemónicos, tanto en estructura, como en su pedagogía y contenido. En otras narrativas, la crítica a la EF apunta a su desvalorización dentro del currículum escolar, cuyo valor educativo en la formación del alumnado de Primaria se cuestiona en favor de otras asignaturas consideradas más académicas.

Creo que la EF es una asignatura secundaria, no tan importante como matemáticas, lengua o ciencia. [...] P.e., suspender EF no es un problema tan grande como tener dificultades en inglés. Estoy de acuerdo con el estereotipo de que la EF es una asignatura "maría". No quiero decir que la EF no importe, pero preferiría que mis estudiantes se centraran en otras asignaturas (M132).

De esta forma, la VS ejercida sobre este alumnado en la EF recibida se hace extensiva a sus proyecciones futuras como docentes, ya sea como sujetos o como agentes de VS. 
Por un lado, pueden no valorar la contribución educativa de la asignatura y autoexcluirse del campo de la EF, adscribiéndose en cambio a otros campos más afines a sus disposiciones. Este profesorado también podría ser agente de VS si no deconstruye la concepción de cuerpo valorado que legitiman los discursos del rendimiento vigentes.

\section{CONCLUSIONES}

En este estudio las experiencias negativas de la EF cursada por aspirantes a profesorado se adscriben poderosamente a los discursos hegemónicos del rendimiento, con orientación androcéntrica. La VS se ejerce porque este alumnado carece de las disposiciones que estos discursos reconocen, valoran y perpetúan (HAY; LISAHUNTER, 2006) y que el profesorado y otro alumnado exitoso legitiman, reproduciéndose unas relaciones de poder a las que el alumnado es sumiso. Esta VS está además institucionalizada dado el carácter oficial, obligatorio y evaluable de la asignatura (BELTRÁN-CARRILLO et al., 2012).

Los resultados también ilustran cómo la propia profesión de profesorado de EF sigue perpetuándose en la persistencia de la dominación masculina. Es una VS que autoexcluye del campo de la EF a las chicas y los chicos que no encajan en el modelo hegemónico, y en consecuencia, este alumnado no se plantea la EF como opción profesional. Precisamente este tipo de estudiantes que han vivido la marginación, podrían ser los verdaderos agentes del cambio ya que están especialmente sensibilizados hacia otras formas de hacer EF.

Este estudio apunta a la necesidad de una reestructuración del propio campo de la EF que redefina las disposiciones y el cuerpo valorado en la asignatura y legitime otras alternativas instauradas en los discursos de la participación, de forma que la EF sea realmente una práctica democrática que dé respuesta a la diversidad y la individualidad. La formación del profesorado debería orientarse en consecuencia al desarrollo de la enseñanza dentro de un marco ético y moral que se centre en dar respuesta al sujeto, a su identidad diferencial, tomando como punto de partida el reconocimiento de la VS presente en el carácter arbitrario de la selección de las prácticas que la EF escolar oficial considera como supuestamente neutras y basadas en el conocimiento "verdadero" (VICENTE, 2013).

Las prácticas reflexivas, a partir de las narrativas de los estudiantes, son fundamentales en la formación del profesorado para ayudarles a entender y tratar de cambiar las expectativas, creencias y valores que configuran el campo de la EF y la VS que sigue perpetuándose. Pero para ello, la propia formación debería dotarles de las herramientas cognitivas y emocionales que les permitan estructurar sus narrativas de la EF de forma radicalmente diferente.

\section{REFERENCIAS}

AZZARITO, Laura.The Panopticon of physical education: pretty, active and ideally white. Physical Education and Sport Pedagogy, v.14, n.1, p.19-39,2009. doi: 10.1080/17408980701712106.

BARBERO, Juan Ignacio. Capital(es) corporal(es) que configuran las corrientes y/o contenidos de la Educación Física escolar. Ágora para la EF y el Deporte,v.4-5, p. 21-38, 2007. 
BELTRÁN-CARRILLO, Vicente J. et al.. When physical activity participation promotes inactivity: Negative experiences of Spanish adolescents in Physical Education and sport. Youth \& Society, v.44, n.1, 3-27, 2012. doi: $10.1177 / 0044118 \times 10388262$.

BOLÍVAR, Antonio; DOMINGO, Jesús; FERNÁNDEZ, Manuel. La investigación biográficonarrativa en educación: Enfoque y metodología. Madrid: La Muralla, 2001.

BOURDIEU, Pierre. La dominación masculina. Barcelona: Anagrama, 2000.

BOURDIEU, Pierre; WACQUANT, Löic. Una invitación a la sociología reflexiva. Buenos Aires: Siglo XXI, 2005

BROWN, David. An economy of gendered practices? Learning to teach physical education from the perspective of Pierre Bourdieu's embodied sociology. Sport, Education and Society, v.10, n.1, p. 3-23, 2005. doi: 10.1080/135733205298785.

COCKBURN, Claudia; CLARKE, Gill. "Everybody's looking at you!": Girls negotiating the "femininity deficit" they incur in physical education. Womens Studies International Forum, v.25, n.6, p. 651-665, 2002.

DEVÍS-DEVÍS, José. La investigación narrativa en la Educación Física y el Deporte. Movimento, v.23, n.1, p.13-24, 2017.doi: http://dx.doi.org/10.22456/1982-8918.71277.

EVANS, John; RICH, Emma; ALLWOOD, Rachel; DAVIES, Brian. Being "able" in a performativa culture: physical education's contribution to a healthy interest in sport. In: WELLARD, I. (Ed.).

Re-thinking gender in youth sport.. London: Routledge, 2007. p. 51-67.

FERNÁNDEZ-BALBOA, Juan Miguel. Transcending masculinities: Linking pedagogy and personhood. In: C. HICKEY, C;FITZCLARENCE, L; MATHEWS, R. (Eds.). Where the boys are: Masculinity, sport and education. Victoria: Deakin University, 1998. p. 121-140.

FERNÁNDEZ, José Manuel. La noción de violencia simbólica en la obra de Pierre Bourdieu: una aproximación crítica. Cuadernos de Trabajo Social, v.18, n.1, p.7-31, 2005.

FISETTE, Jennifer L. 'Are you listening?': adolescent girls voice how they negotiate self-identified barriers to their success and survival in physical education. Physical Education and Sport Pedagogy, v.18, n.2, p.184-203, 2012. doi: 10.1080/17408989.2011.649724.

GARRETT, Robyne. Negotiating a physical identity: girls, bodies and physical education. Sport, Education and Society, v.9, n.2, p.223-237, 2004. doi: 10.1080/1357332042000233958.

GARRETT, Robyne; WRENCH, Alison. Physical experiences: primary student teachers' conceptions of sport and physical education. Physical Education and Sport Pedagogy, v.12, n.1, p.23-42, 2007. doi: 10.1080/17408980601060234.

GLASER, Bernie G.; STRAUSS, Anselm. The discovery of grounded theory: Strategies for qualitative research. Chicago: Aldine, 1967.

GONZÁLEZ PLATE, Lylian; RIVERA, Enrique; TRIGUEROS, Carmen. La interacción social en el contexto del aula de Educación Física. Profesorado: Revista de curriculum y formación del profesorado, v.18, n.2, p. 305-320, 2014.

HAY, Peter J.; HUNTER, Lisa. Please Mr Hay, what are my poss(abilities)?': legitimation of ability through physical education practices. Sport, Education and Society, v.11, n.3, p. 293310, 2006. doi: 10.1080/13573320600813481. 
HAY, Peter J.; MACDONALD, Doune. The gendering of abilities in Senior PE. Physical Education and Sport Pedagogy, v.15, n.3, p.271-285, 2010. doi: 10.1080/17408980903150147.

KIRK, David. Physical Education, Discourse, and Ideology: Bringing the Hidden Curriculum Into View. Quest, v.44, n.1, p.35-56, 1992. doi: 10.1080/00336297.1992.10484040.

LARSSON, Håkan; FAGRELL, Birgitta; REDELIUS, Karin. Queering physical education. Between benevolence towards girls and a tribute to masculinity. Physical Education and Sport Pedagogy, v.14, n.1, p.1-17, 2009. doi: $10.1080 / 17408980701345832$.

LÓPEZ-PASTOR, Victor Manuel et al.Alternative assessment in physical education: a review of international literature. Sport, Education and Society, v.18, n.1, p. 57-76, 2013. doi: 10.1080/13573322.2012.713860.

LÓPEZ PASTOR, Víctor Manuel; GEA FERNÁNDEZ, J. Manuel. Innovación, discurso y racionalidad en educación física. Revisión y prospectiva. Revista Internacional de Medicina y Ciencias de la Actividad Física y el Deporte, v.10, n. 38, p.245-270, 2010.

MONFORTE, Javier; PÉREZ-SAMANIEGO, Víctor. El miedo en Educación Física: una historia reconocible. Movimento, v.23, n.1, p.85-100, 2017. doi: http://dx.doi.org/10.22456/1982$\underline{8918.71272 .}$.

MORGAN, Phillip; BOURKE, Sid. Non-specialist teachers' confidence to teach PE: the nature and influence of personal school experiences in PE. Physical Education and Sport Pedagogy,

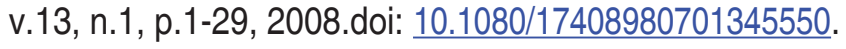

PAECHTER, Carrie. Power, Bodies and Identity: How different forms of physical education construct varying masculinities and femininities in secondary schools. Sex Education, v.3, n.1, p.47-59, 2003.doi: 10.1080/1468181032000052153.

REDELIUS, Karin; FAGRELL, Birgitta; LARSSON, Håkan. Symbolic capital in physical education and health: to be, to do or to know? That is the gendered question. Sport, Education and Society, v.14, n.2, p.245-260, 2009. doi: 10.1080/13573320902809195.

TINNING, Richard. Discursos que orientan el campo del movimiento humano y el problema de la formación del profesorado. Revista de Educación, n. 311, p.123-134, 1996.

TINNING, Richard. Pedagogy and human movement. London: Routledge, 2010.

VICENTE, Manuel. Crítica de la educación física y Educación Física Crítica en España. Estado (crítico) de la cuestión. Movimento, v.19, n.1, p.309-329, 2013. doi: http://dx.doi. org/10.22456/1982-8918.35661.

WRENCH, Alison; GARRETT, Robyne. Pleasure and pain: Experiences of fitness testing. European Physical Education Review, v.14, n.3, p.325-346, 2008. doi: $\underline{10.1177 / 1356336 \times 08095669}$.

WRIGHT, Jan; BURROWS, Lisette. Re-conceiving ability in physical education: A social analysis. Sport, Education and Society, v.11, n.3, p.275-291, 2006. doi: 10.1080/13573320600813440.

Agradecimientos:

Le agradecemos a Ana Rey-Cao los comentarios y sugerencias realizados sobre un borrador previo. 